\title{
The Set of Diagnostics for the First Operation Campaign of the Wendelstein 7-X Stellarator
}

Ralf König*, ${ }^{1}$

E-mail: Ralf.Koenig@ipp.mpg.de

J. Baldzuhn ${ }^{1}$, W. Biel ${ }^{2}$, C. Biedermann ${ }^{1}$, H. S. Bosch ${ }^{1}$, S. Bozhenkov ${ }^{1}$, T. Bräuer ${ }^{1}$, B. Brotas de Carvalho ${ }^{3}$, R. Burhenn ${ }^{1}$, B. Buttenschön ${ }^{1}$, G. Cseh $^{4}$, A. Czarnecka ${ }^{5}$, M. Endler ${ }^{1}$, V. Erckmann ${ }^{1}$, T. Estrada ${ }^{6}$, J. Geiger ${ }^{1}$, O. Grulke ${ }^{1}$, D. Hartmann ${ }^{1}$, D. Hathiramani $^{1}$, M.Hirsch ${ }^{1}$, S. Jabłonski ${ }^{5}$, M. Jakubowski ${ }^{1}$, J. Kaczmarczyk $^{5}$, T. Klinger $^{1}$, S. Klose ${ }^{1}$, G. Kocsis ${ }^{4}$, P. Kornejew ${ }^{1}$, A. Krämer-Flecken ${ }^{2}$, T. Kremeyer ${ }^{7}$, M. Krychowiak $^{1}$, M. Kubkowska ${ }^{5}$, A. Langenberg ${ }^{1}$, H. P. Laqua ${ }^{1}$, M. Laux ${ }^{1}$, Y. Liang ${ }^{2}$, A. Lorenz ${ }^{1}$, A., O. Marchuk ${ }^{2}$, V. Moncada ${ }^{8}$, O. Neubauer ${ }^{2}$, U. Neuner ${ }^{1}$, J. W. Oosterbeek $^{9}$, M. Otte ${ }^{1}$, N. Pablant ${ }^{10}$, E. Pasch ${ }^{1}$, T. S. Pedersen ${ }^{1}$, K. Rahbarnia ${ }^{1}$, L. Ryc $^{5}$, O. Schmitz ${ }^{7}$, W. Schneider ${ }^{1}$, H. Schuhmacher ${ }^{11}$, B. Schweer ${ }^{2}$, T. Stange ${ }^{1}$, H. Thomsen $^{1}$, J.-M. Travere ${ }^{8}$, T. Szepesi ${ }^{4}$, U. Wenzel ${ }^{1}$, A. Werner ${ }^{1}$, B. Wiegel ${ }^{11}$, T. Windisch $^{1}$, R. Wolf ${ }^{1}$, G. A. Wurden ${ }^{12}$, D. Zhang ${ }^{1}$, A. Zimbal ${ }^{11}$, S. Zoletnik ${ }^{4}$ and the W7-X Team

${ }^{1}$ Max Planck Inst. for Plasma Physics,17491 Greifswald, Germany,

${ }^{2}$ Institute of Energy- and Climate Research, Forschungszentrum Jülich GmbH, D-52425 Jülich, Germany

${ }^{3}$ Instituto de Plasmas e Fusao Nuclear Instituto Superior Tecnico, Lisbon, Portugal

${ }^{4}$ Wigner RCP, RMI, Konkoly Thege 29-33, H-1121 Budapest, Hungary

${ }^{5}$ IFPiLM, Hery Street 23, 01-497 Warsaw, Poland

${ }^{6}$ Laboratorio Nacional de Fusi'on, CIEMAT, Avenida Complutense, Madrid, Spain

${ }^{7}$ Univ. of Wisconsin, Dept. of Engineering Physics, 1500 Engineering Drive, Madison, WI 53706

${ }^{8}$ CEA, IRFM, F-13108 Saint-Paul-lez-Durance, France

${ }^{9}$ Eindhoven University of Technology, P.O. Box 513, 5600 MB Eindhoven, The Netherlands

${ }^{10}$ Princeton Plasma Physics Laboratory, Princeton, New Jersey 08543, USA

${ }^{11}$ Physikalisch-Technische Bundesanstalt, Bundesallee 100, 38116 Braunschweig, Germany

${ }^{12}$ Los Alamos National Laboratory, Los Alamos, NM 87544, USA

ABSTRACT: Wendelstein 7-X (W7-X) is a large optimized stellarator $\left(\mathrm{B}=2.5 \mathrm{~T}, \mathrm{~V}=30 \mathrm{~m}^{3}\right)$ aiming at demonstrating the reactor relevance of the optimized stellarators. In summer this year (2015) W7-X will begin its first operation phase (OP1.1) with five inertially cooled inboard limiters made of graphite. Assuming the heat loads can be spread out evenly between the limiters, 1 second discharges at $2 \mathrm{MW}$ of heating power could be run in OP1.1. The diagnostics available for this first operation phase, including some special limiter diagnostics, and their capabilities are being presented.

This paper will be published in the special $1^{\text {st }}$ ECPD issue of JINST.

First EPS Conference on Plasma Diagnostics - $1^{\text {st }}$ ECPD

14-17 April 2015,

Villa Mondragone , Frascati (Rome) Italy

*Speaker 